\title{
Dissipative Delay-Feedback Control for Nonlinear Stochastic Systems with Time-Varying Delay
}

\author{
Guici Chen, ${ }^{1,2}$ Jianzhong Zhou, ${ }^{1}$ and Yongchuan Zhang ${ }^{1}$ \\ ${ }^{1}$ School of Hydropower \& Information Engineering, Huazhong University of Science \& Technology, Wuhan 430074, China \\ ${ }^{2}$ Hubei Province Key Laboratory of Systems Science in Metallurgical Process, Wuhan University of Science er Technology, \\ Wuhan 430081, China
}

Correspondence should be addressed to Guici Chen; gcichen@163.com

Received 26 January 2014; Accepted 2 May 2014; Published 21 May 2014

Academic Editor: Weihai Zhang

Copyright (C) 2014 Guici Chen et al. This is an open access article distributed under the Creative Commons Attribution License, which permits unrestricted use, distribution, and reproduction in any medium, provided the original work is properly cited.

\begin{abstract}
The dissipative delay-feedback control problems for nonlinear stochastic delay systems (NSDSs) based on dissipativity analysis are studied in this paper. Based on the Lyapunov stability theory and stochastic analysis technique, both delay-independent and delaydependent dissipativity criteria are established as linear matrix inequalities- (LMIs-) based feasibility tests. The obtained results in this paper for the nominal systems include the available results on $H_{\infty}$ approach and passivity for stochastic delay systems as special cases. The delay-dependent feedback controller is designed by considering the relationship among the time-varying delay, its lower and upper bound, and its differential without ignoring any terms, which effectively reduces the conservative. A numerical example is given to illustrate the theoretical developments.
\end{abstract}

\section{Introduction}

The stochastic differential systems appear as a natural description of many observed phenomena of real world, which have been come to play an important role in many fields including population dynamics, macroeconomics, chemical reactor control, communication network, image processes, and mobile robot localization. Therefore, the stability and stabilizability of nonlinear stochastic differential systems affine in the control have been studied in the past years by means of the stochastic Lyapunov theory [1-4]. As we all know, time delay in a control loop is one of the main sources of instability, oscillation, and poor performance and naturally encountered in a number of engineering control problems and physical systems. Therefore, time-delay systems $[5,6]$ and stochastic time-delay systems [7-12] have attracted many researchers' attention and have been extensively studied. The problems include stability analysis $[5,6]$, stabilization problems $[7,8]$, and robust controller design [9-12].

On the other hand, studying the dissipativity analysis and synthesis problems has a strong motivation due to their simplicity and effectiveness in dealing with robust and nonlinear systems. Since the notation of dissipative dynamical system was introduced by Willems [13], dissipative systems have been of particular interest to researchers in areas of systems, circuits, networks and control, and so forth. Moreover, passivity of a certain system in a feedback interconnected system will ensure the overall stability of that feedback system if uncertainties or nonlinearities can be characterized by a strict passive system. Hence, dissipative theory has wide ranging implications and applications in control theory. For instance, dissipativity was crucially used in the stability analysis of nonlinear system [14]; the theory of dissipative systems generalizes basic tools including the passivity theory, bounded real lemma, Kalman Yakubovich lemma, and the circle criterion [15]. Among the relevant topics are the dissipativity analysis and synthesis for time-delay systems $[16,17]$. These results show that the dissipativity-based methods are highly effective in design the robust controller.

Due to what is above mentioned, we believe that time delay is often harmful factor of systems. However, time delay is also surprising since plenty of studies have shown that time delay can also benefit the control, such as time-delay control. As mentioned previously, many results have been 
published about the control of systems with state delays but without input delays, which is called memoryless controllers, or to more general, memoryless controllers only includes an instantaneous feedback term. The time-delay control is an approach which gives a small delay $h$ in the controller design, so as to reduce the effect of instability factor and exogenous disturbance. See, for example [18-20], and the references therein. Rather than adjusting control gains or identifying model parameters, its essential idea is to use past observations regarding both the control input and system response, which is an open problem now. In this paper, dissipative delayfeedback control problems for nonlinear stochastic systems with time-varying delay are studied based on dissipativity. The delay-dependent feedback controller is designed by considering the relationship among the time-varying delay, its lower and upper bound, and its differential without ignoring any terms, which effectively reduces the conservative.

\section{Problems Statement and Preliminaries}

In this paper, we consider the following nonlinear stochastic delayed systems (NSDSs) defined on a probability space $(\Omega, \mathscr{F}, \mathscr{P})$ :

$$
\begin{aligned}
\mathrm{d} x(t)=\{ & F(t, x(t), x(t-\tau(t)))+B u(t, t-\tau(t)) \\
& +D v(t)\} \mathrm{d} t \\
+ & G(t, x(t), x(t-\tau(t))) \mathrm{d} \omega(t), \\
z & (t)=C x(t)+C_{d} x(t-\tau(t)), \\
& x(t)=\varphi(t), \quad t \in\left[-\tau_{2}, 0\right),
\end{aligned}
$$

where $x(t) \in \mathbb{R}^{n}$ is the state vector; $u(t, t-\tau(t)) \in \mathbb{R}^{m}$ is the control input, which depends on not only the real time but also the delay; we call the controller memory controller; $z(t) \in \mathbb{R}^{q}$ is the control output; $v(t) \in \mathbb{R}^{p}$ is the exogenous disturbance input, which satisfies $v(t) \in L_{2}\left([0, \infty), \mathbb{R}^{p}\right)$, where $L_{2}\left([0, \infty), \mathbb{R}^{p}\right)$ is the space of nonanticipatory squaresummable stochastic process with respect to $\left(\mathscr{F}_{t}\right)_{t>0}$ with the following norm: $\|v(t)\|_{2}^{2}=\mathbb{E} \int_{0}^{\infty}\|v(t)\|^{2} \mathrm{~d} t . \omega(t) \in \mathbb{R}^{l}$ is a scalar Brownian motion defined on a complete probability space $(\Omega, \mathscr{F}, P)$ with $\mathbb{E}[\mathrm{d} \omega(t)]=0, \mathbb{E}\left[\mathrm{d}^{2} \omega(t)\right]=\mathrm{d} t$.

In the sequel, we seek to study the problems of dissipative analysis and delay-feedback control for the two cases of time delay.

Case 1. Time delay is a constant $\tau$.

Case 2. $\tau(t)$ is the time-varying delay, which is a differential function satisfying

$$
0 \leq \tau_{1} \leq \tau(t) \leq \tau_{2}, \quad \dot{\tau}(t) \leq \tau_{d} \leq 1,
$$

where $\tau_{1}, \tau_{2}$, and $\tau_{d}$ are nonnegative constants.

Remark 1. Obviously, when $\tau_{d}=0, \tau(t)=\tau_{1}=\tau_{2}$, that means the time delay is a constant; this case has been extensively studied. On the other hand, the time-varying delay $\tau(t) \geq \tau_{1}$; here $\tau_{1}$ is equal to or greater than 0 , which has less conservativeness than $\tau(t)>0$.
Assumption 2. $F(\cdot, \cdot, \cdot)$ is a nonlinear vector function which can be decomposed as follows:

$$
\begin{aligned}
F(t, x(t), x(t-\tau(t))) & \\
= & A_{0} x(t)+f(t, x(t)) \\
& \quad+A_{d} x(t-\tau(t))+f_{d}(t, x(t-\tau(t))),
\end{aligned}
$$

where $f, f_{d}$ are vector-valued functions; we assume

$$
\begin{gathered}
\|f(t, x(t))\| \leq \beta\|x(t)\|, \\
\left\|f_{d}(t, x(t-\tau(t)))\right\| \leq \beta_{d}\|x(t-\tau(t))\|,
\end{gathered}
$$

where $\beta, \beta_{d}$ are known real positive constants.

Obviously, we know that

$$
f(0,0)=0, \quad f_{d}(0,0)=0 .
$$

Equivalently stated, condition (4) implies that there exists a scalar $\kappa>0$ such that

$$
\kappa\left(\beta^{2} x^{T}(t) x(t)-f^{T}(t, x(t)) f(t, x(t))\right) \geq 0 .
$$

Similarly, condition (5) implies that there exists a scalar $\kappa_{d}>0$ such that

$$
\begin{aligned}
\kappa_{d}( & \beta_{d}^{2} x^{T}(t-\tau(t)) x(t-\tau(t)) \\
& \left.\quad-f_{d}^{T}(t, x(t-\tau(t))) f_{d}(t, x(t-\tau(t)))\right) \geq 0 .
\end{aligned}
$$

Assumption 3. $G(\cdot, \cdot, \cdot)$ is a nonlinear vector function which satisfies

$$
\begin{gathered}
\operatorname{Trace}\left(G^{T}(t, x(t), x(t-\tau(t))) G(t, x(t), x(t-\tau(t)))\right) \\
\leq x^{T}(t) \Theta_{1}^{T} \Theta_{1} x(t)+x^{T}(t-\tau(t)) \Theta_{2}^{T} \Theta_{2} x(t-\tau(t)),
\end{gathered}
$$

where $\Theta_{1}, \Theta_{2}$ are known real matrices.

Hence, nonlinear stochastic delay systems (NSDSs) (1) can be rewritten as

$$
\begin{aligned}
& \mathrm{d} x(t)=\left\{A x(t)+f(t, x(t))+A_{d} x(t-\tau(t))\right. \\
&+f_{d}(t, x(t-\tau(t))) \\
&+B u(t, t-\tau(t))+D v(t)\} \mathrm{d} t \\
&+G(t, x(t), x(t-\tau(t))) \mathrm{d} \omega(t), \\
& z(t)=C x(t)+C_{d} x(t-\tau(t)), \\
& x(t)=\varphi(t), \quad t \in\left[-\tau_{2}, 0\right) .
\end{aligned}
$$

Definition 4 (see [21]). Given matrices $Q^{T}=Q \leq 0, R^{T}=$ $R \geq 0$, and $S$, nonlinear stochastic delay systems (NSDSs) (10) are called $(Q, S, R)$-dissipative if, for some real function $\eta(\cdot)$, $\eta(0)=0$,

$$
\begin{aligned}
& \mathbb{E} \int_{0}^{T}\left[z^{T}(s) Q z(s)+2 v^{T}(s) S z(s)+v^{T}(s) R v(s)\right] \mathrm{d} s \\
& \quad+\eta\left(x_{0}\right) \geq 0, \quad \forall T \geq 0 .
\end{aligned}
$$


Furthermore, if, for a scalar $\alpha>0$,

$$
\begin{gathered}
\mathbb{E} \int_{0}^{T}\left[z^{T}(s) Q z(s)+2 v^{T}(s) S z(s)+v^{T}(s) R v(s)\right] \mathrm{d} s \\
+\eta\left(x_{0}\right) \geq \mathbb{E} \int_{0}^{T} \alpha v^{T}(s) v(s) \mathrm{d} s, \quad \forall T \geq 0,
\end{gathered}
$$

NSDSs (10) are called strictly $(Q, S, R)$-dissipative.

Lemma 5 (see [22]). Given three constant matrices $S_{1}, S_{2}$, and $S_{3}$, where $S_{3}=S_{3}^{T}<0$ and $S_{1}=S_{1}^{T}<0$, then $S_{1}-S_{2}^{T} S_{3}^{-1} S_{2}<0$ holds if and only if $\left(\begin{array}{cc}S_{1} & S_{2} \\ S_{2}^{T} & S_{3}\end{array}\right)<0$ or $\left(\begin{array}{cc}S_{3} & S_{2} \\ S_{2}^{T} & S_{1}\end{array}\right)<0$.

Lemma 6 (see [23]). For given positive symmetric matrix $M=$ $M^{T}>0$, two scalars $a$ and $b$ satisfying $a<b$, and vector function $x(t):[a, b] \rightarrow \mathbb{R}^{n}$, then

$$
\left[\int_{a}^{b} x(s) d s\right]^{T} M\left[\int_{a}^{b} x(s) d s\right] \leq(b-a) \int_{a}^{b} x^{T}(s) M x(s) d s .
$$

\section{Dissipativity Analysis for NSDSs}

In this section, our primary purpose is to develope delayindependent and delay-dependent stochastically stability and dissipativity criteria for NSDSs (10) based on Definition 4.

3.1. Delay-Independent Dissipativity. In this sequel, we consider the time delay as unknown constant pertaining to Case 1 and hence the results developed hereinafter will be independent of the size of delay. Without regard to the control input, setting $u(t, t-\tau(t))=0$, then (10) can be rewritten as

$$
\begin{aligned}
& \mathrm{d} x(t)=\left\{A x(t)+f(t, x(t))+A_{d} x(t-\tau)\right. \\
&\left.+f_{d}(t, x(t-\tau))+D v(t)\right\} \mathrm{d} t \\
&+G(t, x(t), x(t-\tau)) \mathrm{d} \omega(t), \\
& z(t)=C x(t)+C_{d} x(t-\tau), \\
& x(t)=\varphi(t), \quad t \in[-\tau, 0) .
\end{aligned}
$$

Theorem 7. Consider the NSDSs (14). Given some scalars $\alpha>$ $0, \beta>0$, and $\beta_{d}>0$ and matrices $Q=Q^{T} \leq 0, R=R^{T}>0$, and $S$, suppose there exist matrices $P=P^{T}>0, W=W^{T}>0$ and positive scalars $\kappa>0, \kappa_{d}>0$ such that the following LMI holds:

$$
\left(\begin{array}{cccccc}
\Sigma_{1} & P A_{d} & \Sigma_{2} & P & P & C^{T} Q \\
* & \Sigma_{3} & -C_{d}^{T} S & 0 & 0 & C_{d}^{T} Q \\
* & * & -R_{\alpha} & 0 & 0 & 0 \\
* & * & * & -\kappa I & 0 & 0 \\
* & * & * & * & -\kappa_{d} I & 0 \\
* & * & * & * & * & Q
\end{array}\right)<0
$$

then the NSDSs (14) are strictly $(Q, S, R)$-dissipative independent of delay, where $\Sigma_{1}=A^{T} P+P A+W+\Theta_{1}^{T} P \Theta_{1}+\kappa \beta^{2} I$, $\Sigma_{2}=P D-C^{T} S, \Sigma_{3}=\Theta_{2}^{T} P \Theta_{2}-W+\kappa_{d} \beta_{d}^{2} I$, and $R_{\alpha}=(R-\alpha I)$.
Proof. At first we introduce the following Lyapunov-Krasovskii functional (LKF):

$$
V\left(x_{t}\right)=x^{T}(t) P x(t)+\int_{t-\tau}^{t} x^{T}(s) W x(s) \mathrm{d} s .
$$

Evaluating the Itô derivative of $V\left(x_{t}\right)$ along the solution of NSDSs (14), we have

$$
\begin{aligned}
\mathscr{L} V\left(x_{t}\right)= & 2 x^{T} P\left[A x(t)+f(t, x(t))+A_{d} x(t-\tau)\right. \\
& \left.+f_{d}(t, x(t-\tau))+D v(t)\right] \\
& +x^{T} W x-x^{T}(t-\tau) W x(t-\tau) \\
& +G^{T}(t, x(t), x(t-\tau)) P G(t, x(t), x(t-\tau)) .
\end{aligned}
$$

Noting (7)-(9), we obtain

$$
\begin{aligned}
& \mathscr{L} V\left(x_{t}\right) \\
& \leq \quad \mathscr{L} V\left(x_{t}\right) \\
& \quad+\kappa\left(\beta^{2} x^{T}(t) x(t)-f^{T}(t, x(t)) f(t, x(t))\right) \\
& \quad+\kappa_{d}\left(\beta_{d}^{2} x^{T}(t-\tau(t)) x(t-\tau(t))\right. \\
& \quad-f_{d}^{T}(t, x(t-\tau(t))) \\
& \left.\quad \times f_{d}(t, x(t-\tau(t)))\right) \\
& \leq \xi^{T} \Sigma \xi,
\end{aligned}
$$

where

$$
\begin{gathered}
\xi=\left(x^{T}(t), x^{T}(t-\tau), v^{T}(t), f^{T}(t, x(t)),\right. \\
\left.f_{d}^{T}(t, x(t-\tau))\right)^{T}, \\
\Sigma=\left(\begin{array}{ccccc}
\Sigma_{1} & P A_{d} & P D & P & P \\
* & \Sigma_{3} & 0 & 0 & 0 \\
* & * & 0 & 0 & 0 \\
* & * & * & -\kappa I & 0 \\
* & * & * & * & -k_{d} I
\end{array}\right) .
\end{gathered}
$$

Hence, we have

$$
\begin{gathered}
\mathscr{L} V\left(x_{t}\right)-z^{T}(s) Q z(s)-2 v^{T}(s) S z(s) \\
-v^{T}(s)(R-\alpha I) v(s) \leq \xi^{T} \widetilde{\Sigma} \xi
\end{gathered}
$$

where

$$
\tilde{\Sigma}=\Sigma-\left(\begin{array}{ccccc}
C^{T} Q C & C^{T} Q C_{d}^{T} & C^{T} S & 0 & 0 \\
* & C_{d}^{T} Q C_{d} & C_{d}^{T} S & 0 & 0 \\
* & * & (R-\alpha I) & 0 & 0 \\
* & * & * & 0 & 0 \\
* & * & * & * & 0
\end{array}\right)
$$


According to Lemma 5 and applying the congruent transformation, we know that

$$
\begin{gathered}
\mathscr{L} V\left(x_{t}\right)-z^{T}(s) Q z(s)-2 v^{T}(s) S z(s) \\
-v^{T}(s)(R-\alpha I) v(s) \leq 0
\end{gathered}
$$

Then, integrating (22) from 0 to $T$ and taking mathematical expectation, we obtain that (12) holds which completes the proof.

Remark 8. When $Q=-I, S=0$, and $R_{\alpha}=\gamma^{2} I$, strictly $(Q, S, R)$-dissipativity reduces to the $H_{\infty}$ performance level. When $Q=0, S=I$, and $R_{\alpha}=\gamma I$, strictly $(Q, S, R)$ dissipativity reduces to the strictly passivity.

So the following corollaries stand out as special cases.

Corollary 9. Consider the NSDSs (14). Given some scalars $\gamma>$ $0, \beta>0$, and $\beta_{d}>0$, suppose there exist matrices $P=P^{T}>0$, $W=W^{T}>0$ and positive scalars $\kappa>0, \kappa_{d}>0$ such that the following LMI holds:

$$
\left(\begin{array}{cccccc}
\Sigma_{1} & P A & P D & P & P & -C^{T} \\
* & \Sigma_{3} & 0 & 0 & 0 & -C_{d}^{T} \\
* & * & -\gamma^{2} I & 0 & 0 & 0 \\
* & * & * & -\kappa I & 0 & 0 \\
* & * & * & * & -\kappa_{d} I & 0 \\
* & * & * & * & * & -I
\end{array}\right)<0
$$

then the NSDSs (14) are stochastically asymptotically stable and independent of delay with disturbance level $\gamma$.

Corollary 10. Consider the NSDSs (14). Given some scalars $\gamma>0, \beta>0$, and $\beta_{d}>0$, suppose there exist matrices $P=P^{T}>0, W=W^{T}>0$ and positive scalars $\kappa>0, \kappa_{d}>0$ such that the following LMI holds:

$$
\left(\begin{array}{ccccc}
\Sigma_{1} & P A & P D-C^{T} & P & P \\
* & \Sigma_{3} & -C_{d}^{T} & 0 & 0 \\
* & * & -\gamma I & 0 & 0 \\
* & * & * & -\kappa I & 0 \\
* & * & * & * & -\kappa_{d} I
\end{array}\right)<0
$$

then the NSDSs (14) are strictly passive independent of delay.

3.2. Delay-Dependent Dissipativity. We now direct attention to the type of dissipativity which depends on the time-varying delay, which pertains to Case 2. Without consideration of the control input, and defining a new state variable

$$
\begin{aligned}
y(t)= & A x(t)+f(t, x(t))+A_{d} x(t-\tau(t)) \\
& +f_{d}(t, x(t-\tau(t)))+D v(t),
\end{aligned}
$$

then, the NSDSs (10) can be rewritten as

$$
\begin{gathered}
\mathrm{d} x(t)=y(t) \mathrm{d} t+G(t, x(t), x(t-\tau(t))) \mathrm{d} \omega(t), \\
z(t)=C x(t)+C_{d} x(t-\tau(t)), \\
x(t)=\varphi(t), \quad t \in\left[-\tau_{2}, 0\right) .
\end{gathered}
$$

Theorem 11. Consider the NSDSs (26). For the given scalars $\alpha>0, \tau_{2}>\tau_{1} \geq 0, \tau_{d}>0, \beta>0$, and $\beta_{d}>0$, the NSDSs (26) are strictly $(Q, S, R)$-dissipative for all time-varying delays if there exist symmetric positive-definite matrices $P, V_{1}, V_{2}, V_{3}$, $W_{1}, W_{2}, Z_{1}$, and $Z_{2}$; any appropriately dimensioned matrices $N, M, F$, and $H$; and two positive scalars $\kappa>0, \kappa_{d}>0$ such that the following LMIs hold:

$$
\begin{gathered}
\left(\begin{array}{cc}
\Xi & -\tau_{2} \widehat{M} \\
* & -\tau_{2} W_{1}
\end{array}\right)<0, \\
\left(\begin{array}{cc}
\Xi & -\left(\tau_{2}-\tau_{1}\right) \widehat{N} \\
* & -\left(\tau_{2}-\tau_{1}\right)\left(W_{1}+W_{2}\right)
\end{array}\right)<0, \\
\left(\begin{array}{ll}
\Xi & -\left(\tau_{2}-\tau_{1}\right) \widehat{F} \\
* & -\left(\tau_{2}-\tau_{1}\right) W_{2}
\end{array}\right)<0,
\end{gathered}
$$

where

$$
\begin{aligned}
& \Xi=\left(\begin{array}{cccccc}
\Xi_{11} & \Xi_{12} & \Xi_{13} & \Xi_{14} & A^{T} H & \Xi_{16} \\
* & \Xi_{22} & -C_{d}^{T} S & 0 & A_{d}^{T} H & \Xi_{26} \\
* & * & -R_{\alpha} & 0 & D^{T} H & 0 \\
* & * & * & \Xi_{44} & \Xi_{45} & 0 \\
* & * & * & * & \Xi_{55} & 0 \\
* & * & * & * & * & \Xi_{66}
\end{array}\right), \\
& \Xi_{11}=P A+A^{T} P+V_{1}+V_{2}+V_{3}+\tau_{2} Z_{1} \\
& +\left(\tau_{2}-\tau_{1}\right) Z_{2}+M+M^{T}+\kappa \beta^{2} I \\
& \Xi_{12}=N-F-M+P A_{d}, \quad \Xi_{13}=P D-C^{T} S, \\
& \Xi_{14}=\left(\begin{array}{llll}
F & -N & P & P
\end{array}\right), \\
& \Xi_{16}=\left(\begin{array}{lll}
C^{T} Q & \Theta_{1} P & 0
\end{array}\right), \quad \Xi_{22}=-\left(1-\tau_{d}\right) V_{2}+\kappa_{d} \beta_{d}^{2} I, \\
& \Xi_{26}=\left(\begin{array}{lll}
C_{d}^{T} Q & 0 & \Theta_{2} P
\end{array}\right) \\
& \Xi_{44}=\operatorname{diag}\left\{-V_{1},-V_{3},-\kappa I,-\kappa_{d} I\right\}, \\
& \Xi_{55}=\tau_{2} W_{1}+\left(\tau_{2}-\tau_{1}\right) W_{2}-H-H^{T}, \\
& \Xi_{66}=\operatorname{diag}\{Q,-P,-P\}, \quad \Xi_{45}=\left(\begin{array}{llll}
0 & 0 & H^{T} & H^{T}
\end{array}\right)^{T}, \\
& \widehat{N}=\left(N^{T}, 0_{1 \times 10}\right)^{T}, \quad \widehat{M}=\left(M^{T}, 0_{1 \times 10}\right)^{T}, \\
& \widehat{F}=\left(F^{T}, 0_{1 \times 10}\right)^{T} .
\end{aligned}
$$

Proof. We construct a LKF as follows:

$$
V\left(x_{t}\right)=V_{1}\left(x_{t}\right)+V_{2}\left(x_{t}\right)+V_{3}\left(x_{t}\right)+V_{4}\left(x_{t}\right),
$$


where

$$
\begin{gathered}
V_{1}\left(x_{t}\right)=x^{T}(t) P x(t), \\
V_{2}\left(x_{t}\right)=\int_{t-\tau_{1}}^{t} x^{T}(s) V_{1} x(s) \mathrm{d} s+\int_{t-\tau(t)}^{t} x^{T}(s) V_{2} x(s) \mathrm{d} s \\
+\int_{t-\tau_{2}}^{t} x^{T}(s) V_{3} x(s) \mathrm{d} s, \\
V_{3}\left(x_{t}\right)=\int_{-\tau_{2}}^{0} \int_{t+\theta}^{t} y^{T}(s) W_{1} y(s) \mathrm{d} s \\
+\int_{-\tau_{2}}^{-\tau_{1}} \int_{t+\theta}^{t} y^{T}(s) W_{2} y(s) \mathrm{d} s, \\
V_{4}\left(x_{t}\right)=\int_{-\tau_{2}}^{0} \int_{t+\theta}^{t} x^{T}(s) Z_{1} x(s) \mathrm{d} s \\
+\int_{-\tau_{2}}^{-\tau_{1}} \int_{t+\theta}^{t} x^{T}(s) Z_{2} x(s) \mathrm{d} s .
\end{gathered}
$$

Then, the weak infinitesimal operator $\mathscr{L}$ of the stochastic process $x_{t}$ along the evolution of $V\left(x_{t}\right)$ is given by

$$
\mathscr{L} V\left(x_{t}\right)=\mathscr{L} V_{1}\left(x_{t}\right)+\mathscr{L} V_{2}\left(x_{t}\right)+\mathscr{L} V_{3}\left(x_{t}\right)+\mathscr{L} V_{4}\left(x_{t}\right)
$$

where

$$
\begin{aligned}
\mathscr{L} V_{1}\left(x_{t}\right) \leq & 2 x^{T}(t) P[A x(t)+f(t, x(t)) \\
& +A_{d} x(t-\tau(t)) \\
& \left.+f_{d}(t, x(t-\tau(t)))+D v(t)\right] \\
& +x^{T}(t) \Theta_{1}^{T} P \Theta_{1} x(t) \\
& +x(t-\tau(t)) \Theta_{2}^{T} P \Theta_{2} x(t-\tau(t)) \\
\mathscr{L} V_{2}\left(x_{t}\right) \leq & x^{T}(t)\left(V_{1}+V_{2}+V_{3}\right) x(t) \\
& -x^{T}\left(t-\tau_{1}\right) V_{1} x\left(t-\tau_{1}\right) \\
& -x^{T}\left(t-\tau_{2}\right) V_{3} x\left(t-\tau_{2}\right) \\
& -x^{T}(t-\tau(t))\left(1-\tau_{d}\right) V_{2} x(t-\tau(t)) \\
\mathscr{L} V_{3}\left(x_{t}\right)= & \tau_{2} y^{T}(t) W_{1} y(t) \\
& -\int_{t-\tau_{2}}^{t} y^{T}(s) W_{1} y(s) \mathrm{d} s \\
& +\left(\tau_{2}-\tau_{1}\right) y^{T}(t) W_{2} y(t) \\
& -\int_{t-\tau_{2}}^{t-\tau_{1}} y^{T}(s) W_{2} y(s) \mathrm{d} s
\end{aligned}
$$

By using Lemma 6, we get the following inequality:

$$
\begin{aligned}
\mathscr{L} V_{4}\left(x_{t}\right) \leq & \tau_{2} x^{T}(t) Z_{1} x(t)+\left(\tau_{2}-\tau_{1}\right) x^{T}(t) Z_{2} x(t) \\
& -\frac{1}{\tau_{2}}\left(\int_{t-\tau(t)}^{t} x(s) \mathrm{d} s\right)^{T} Z_{1}\left(\int_{t-\tau(t)}^{t} x(s) \mathrm{d} s\right) \\
& -\frac{1}{\tau_{2}-\tau_{1}}\left(\int_{t-\tau_{2}}^{t-\tau(t)} x(s) \mathrm{d} s\right)^{T} Z_{2}\left(\int_{t-\tau_{2}}^{t-\tau(t)} x(s) \mathrm{d} s\right) \\
& -\frac{1}{\tau_{2}-\tau_{1}}\left(\int_{t-\tau(t)}^{t-\tau_{1}} x(s) \mathrm{d} s\right)^{T} Z_{2}\left(\int_{t-\tau(t)}^{t-\tau_{1}} x(s) \mathrm{d} s\right),
\end{aligned}
$$

setting $\xi(t)=\left(x^{T}(t), x^{T}(t-\tau(t)), v^{T}(t), x^{T}\left(t-\tau_{1}\right), x^{T}(t-\right.$ $\left.\tau_{2}\right), f^{T}(x(t)), f_{d}^{T}(x(t \quad-\quad \tau(t))), y^{T}(t),\left(\int_{t-\tau(t)}^{t} x(s) \mathrm{d} s\right)^{T}$, $\left.\left(\int_{t-\tau_{2}}^{t-\tau(t)} x(s) \mathrm{d} s\right)^{T},\left(\int_{t-\tau(t)}^{t-\tau_{1}} x(s) \mathrm{d} s\right)^{T}\right)^{T}$. And we introduce the following four zero equations:

$$
\begin{aligned}
& 2 x^{T}(t) M\left[x(t)-x(t-\tau(t))-\int_{t-\tau(t)}^{t} \mathrm{~d} x(s)\right]=0, \\
& 2 x^{T}(t) N\left[x(t-\tau(t))-x\left(t-\tau_{2}\right)-\int_{t-\tau_{2}}^{t-\tau(t)} \mathrm{d} x(s)\right]=0, \\
& 2 x^{T}(t) F\left[x\left(t-\tau_{1}\right)-x(t-\tau(t))-\int_{t-\tau(t)}^{t-\tau_{1}} \mathrm{~d} x(s)\right]=0,
\end{aligned}
$$

$$
\begin{aligned}
& 2 y^{T}(t) H[ A x(t)+f(t, x(t))+A_{d} x(t-\tau(t)) \\
&\left.+f_{d}(t, x(t-\tau(t)))+D v(t)-y(t)\right]=0 .
\end{aligned}
$$

Summing up (31)-(39), we obtain

$$
\begin{aligned}
& \mathscr{L} V\left(x_{t}\right) \leq \mathscr{L} V\left(x_{t}\right) \\
&+\kappa\left(\beta^{2} x^{T}(t) x(t)-f^{T}(t, x(t)) f(t, x(t))\right) \\
&+\kappa_{d}\left(\beta_{d}^{2} x^{T}(t-\tau(t)) x(t-\tau(t))\right. \\
&\left.-f_{d}^{T}(t, x(t-\tau(t))) f_{d}(t, x(t-\tau(t)))\right) \\
&+2 x^{T}(t) M[x(t)-x(t-\tau(t)) \\
&\left.-\int_{t-\tau(t)}^{t} y(s) \mathrm{d} s\right] \\
&+2 x^{T}(t) M \int_{t-\tau(t)}^{t} G\left(x_{s}\right) \mathrm{d} \omega(s) \\
& N {\left[x(t-\tau(t))-x\left(t-\tau_{2}\right)\right.} \\
&\left.-\int_{t-\tau_{2}}^{t-\tau(t)} y(s) \mathrm{d} s\right]
\end{aligned}
$$




$$
\begin{gathered}
-2 x^{T}(t) N \int_{t-\tau_{2}}^{t-\tau(t)} G\left(x_{s}\right) \mathrm{d} \omega(s) \\
+2 x^{T}(t) F\left[x\left(t-\tau_{1}\right)-x(t-\tau(t))\right. \\
\left.-\int_{t-\tau(t)}^{t-\tau_{1}} y(s) \mathrm{d} s\right] \\
-2 x^{T}(t) F \int_{t-\tau(t)}^{t-\tau_{1}} G\left(x_{s}\right) \mathrm{d} \omega(s) \\
+2 y^{T}(t) H[A x(t)+f(t, x(t)) \\
+A_{d} x(t-\tau(t)) \\
+f_{d}(t, x(t-\tau(t))) \\
+D v(t)-y(t)]
\end{gathered}
$$

where $M, N, F$, and $H$ are matrices with appropriate dimensions. Hence,

$$
\begin{aligned}
\mathscr{L} V\left(x_{t}\right) & -z^{T}(s) Q z(s)-2 v^{T}(s) S z(s) \\
& -v^{T}(s)(R-\alpha I) v(s) \\
\leq & \xi^{T}(t) \Phi \xi(t)-2 x^{T}(t) M \\
& \times\left[\int_{t-\tau(t)}^{t} y(s) \mathrm{d} s+\int_{t-\tau(t)}^{t} G\left(x_{s}\right) \mathrm{d} \omega(s)\right] \\
& -2 x^{T}(t) N\left[\int_{t-\tau_{2}}^{t-\tau(t)} y(s) \mathrm{d} s+\int_{t-\tau_{2}}^{t-\tau(t)} G\left(x_{s}\right) \mathrm{d} \omega(s)\right] \\
& -2 x^{T}(t) F\left[\int_{t-\tau(t)}^{t-\tau_{1}} y(s) \mathrm{d} s+\int_{t-\tau(t)}^{t-\tau_{1}} G\left(x_{s}\right) \mathrm{d} \omega(s)\right] \\
& -\int_{t-\tau_{2}}^{t} y^{T}(s) W_{1} y(s) \mathrm{d} s-\int_{t-\tau_{2}}^{t-\tau_{1}} y^{T}(s) W_{2} y(s) \mathrm{d} s
\end{aligned}
$$

where

$$
\begin{gathered}
\Phi=\left(\begin{array}{cccccc}
\Phi_{11} & \Phi_{12} & \Phi_{13} & \Phi_{14} & A^{T} H & 0 \\
* & \Phi_{22} & -C_{d}^{T} S & 0 & A_{d}^{T} H & 0 \\
* & * & -R_{\alpha} & 0 & D^{T} H & 0 \\
* & * & * & \Phi_{44} & \Phi_{45} & 0 \\
* & * & * & * & \Phi_{55} & 0 \\
* & * & * & * & * & \Phi_{66}
\end{array}\right), \\
\Phi_{11}=P A+A^{T} P+\Theta_{1}^{T} P \Theta_{1}+V_{1}+V_{2}+V_{3}+\tau_{2} Z_{1} \\
+\left(\tau_{2}-\tau_{1}\right) Z_{2}+M+M^{T}-C^{T} Q C+\kappa \beta^{2} I, \\
\Phi_{12}=N-F-M+P A_{d}-C^{T} Q C_{d}, \quad \Phi_{13}=P D-C^{T} S,
\end{gathered}
$$

$$
\begin{aligned}
& \Phi_{14}=\left(\begin{array}{llll}
F & -N & P & P
\end{array}\right), \\
& \Phi_{22}=\Theta_{2}^{T} P \Theta_{2}-C_{d}^{T} Q C_{d}-\left(1-\tau_{d}\right) V_{2}+\kappa_{d} \beta_{d}^{2} I, \\
& \Phi_{44}=\operatorname{diag}\left\{-V_{1},-V_{3},-\kappa I,-\kappa_{d} I\right\}, \\
& \Phi_{45}=\left(\begin{array}{llll}
0 & 0 & H^{T} & H^{T}
\end{array}\right)^{T}, \\
& \Phi_{55}=\tau_{2} W_{1}+\left(\tau_{2}-\tau_{1}\right) W_{2}-H-H^{T}, \\
& \Phi_{66}=\operatorname{diag}\left\{-\frac{1}{\tau_{2}} Z_{1},-\frac{1}{\tau_{2}-\tau_{1}} Z_{2},-\frac{1}{\tau_{2}-\tau_{1}} Z_{2}\right\} \text {. }
\end{aligned}
$$

So it easy to obtain that

$$
\begin{aligned}
& \mathscr{L} V\left(x_{t}\right)-z^{T}(s) Q z(s)-2 v^{T}(s) S z(s)-v^{T}(s)(R-\alpha I) v(s) \\
& \leq \frac{1}{\tau_{2}} \int_{t-\tau(t)}^{t} \eta^{T}(t, s)\left(\begin{array}{cc}
\Phi & -\tau_{2} \widehat{M} \\
* & -\tau_{2} W_{1}
\end{array}\right) \eta(t, s) \mathrm{d} s \\
& +\frac{1}{\tau_{2}-\tau_{1}} \int_{t-\tau(t)}^{t} \eta^{T}(t, s) \\
& \times\left(\begin{array}{cc}
\Phi & -\left(\tau_{2}-\tau_{1}\right) \widehat{N} \\
* & -\left(\tau_{2}-\tau_{1}\right)\left(W_{1}+W_{2}\right)
\end{array}\right) \eta(t, s) \mathrm{d} s \\
& +\frac{1}{\tau_{2}-\tau_{1}} \int_{t-\tau(t)}^{t} \eta^{T}(t, s)\left(\begin{array}{cc}
\Phi & -\left(\tau_{2}-\tau_{1}\right) \widehat{F} \\
* & -\left(\tau_{2}-\tau_{1}\right) W_{2}
\end{array}\right) \eta(t, s) \mathrm{d} s \\
& -2 x^{T}(t) M \int_{t-\tau(t)}^{t} G\left(x_{s}\right) \mathrm{d} \omega(s) \\
& -2 x^{T}(t) N \int_{t-\tau_{2}}^{t-\tau(t)} G\left(x_{s}\right) \mathrm{d} \omega(s) \\
& -2 x^{T}(t) F \int_{t-\tau(t)}^{t-\tau_{1}} G\left(x_{s}\right) \mathrm{d} \omega(s),
\end{aligned}
$$

where $\eta^{T}(t, s)=\left[\xi^{T}(t), y^{T}(s)\right]$.

By Lemma 5 and applying the congruent transformation to (27), it follows that

$$
\begin{aligned}
\mathscr{L} V\left(x_{t}\right) & -z^{T}(s) Q z(s)-2 v^{T}(s) S z(s) \\
& -v^{T}(s)(R-\alpha I) v(s) \\
\leq & -2 x^{T}(t) M \int_{t-\tau(t)}^{t} G\left(x_{s}\right) \mathrm{d} \omega(s) \\
& -2 x^{T}(t) N \int_{t-\tau_{2}}^{t-\tau(t)} G\left(x_{s}\right) \mathrm{d} \omega(s) \\
& -2 x^{T}(t) F \int_{t-\tau(t)}^{t-\tau_{1}} G\left(x_{s}\right) \mathrm{d} \omega(s) .
\end{aligned}
$$

Then, integrating both sides of (44) from 0 to $T$ and taking mathematical expectation, we obtain that (12) holds, which completes the proof. 
Similarly, as the special case, we can easily obtain the following corollaries.

Corollary 12. Consider the NSDSs (26). For the given scalars $\gamma>0, \tau_{2}>\tau_{1} \geq 0, \tau_{d}>0, \beta>0$, and $\beta_{d}>0$, the NSDSs (26) are stochastically asymptotically stable with $H_{\infty}$ performance level for all time-varying delays if there exist symmetric positive-definite matrices $P, V_{1}, V_{2}, V_{3}, W_{1}, W_{2}, Z_{1}$, and $Z_{2}$; any appropriately dimensioned matrices $N, M, F$, and $H$; and two positive scalars $\kappa>0, \kappa_{d}>0$ such that the following LMIs hold:

$$
\begin{gathered}
\left(\begin{array}{cc}
\Xi & -\tau_{2} \widehat{M} \\
* & -\tau_{2} W_{1}
\end{array}\right)<0, \\
\left(\begin{array}{cc}
\Xi & -\left(\tau_{2}-\tau_{1}\right) \widehat{N} \\
* & -\left(\tau_{2}-\tau_{1}\right)\left(W_{1}+W_{2}\right)
\end{array}\right)<0, \\
\left(\begin{array}{cc}
\Xi & -\left(\tau_{2}-\tau_{1}\right) \widehat{F} \\
* & -\left(\tau_{2}-\tau_{1}\right) W_{2}
\end{array}\right)<0,
\end{gathered}
$$

where

$$
\begin{aligned}
& \Xi=\left(\begin{array}{cccccc}
\Xi_{11} & \Xi_{12} & P D & \Xi_{14} & A^{T} H & \Xi_{16} \\
* & \Xi_{22} & 0 & 0 & A_{d}^{T} H & \Xi_{26} \\
* & * & -\gamma^{2} I & 0 & D^{T} H & 0 \\
* & * & * & \Xi_{44} & \Xi_{45} & 0 \\
* & * & * & * & \Xi_{55} & 0 \\
* & * & * & * & * & \Xi_{66}
\end{array}\right), \\
& \Xi_{16}=\left(\begin{array}{lll}
-C^{T} & \Theta_{1} P & 0
\end{array}\right), \quad \Xi_{26}=\left(\begin{array}{lll}
-C_{d}^{T} & 0 & \Theta_{2} P
\end{array}\right), \\
& \Xi_{66}=\operatorname{diag}\{-I,-P,-P\}, \\
& \Xi_{11}, \Xi_{12}, \Xi_{14}, \Xi_{22}, \Xi_{44}, \Xi_{45}, \Xi_{55},
\end{aligned}
$$

defined in Theorem 11.

Corollary 13. Consider the NSDSs (26). For the given scalars $\gamma>0, \tau_{2}>\tau_{1} \geq 0, \tau_{d}>0, \beta>0$, and $\beta_{d}>0$, the NSDSs (26) are strictly $(Q, S, R)$-passive for all time-varying delays if there exist symmetric positive-definite matrices $P, V_{1}, V_{2}, V_{3}$, $W_{1}, W_{2}, Z_{1}, Z_{2}$; any appropriately dimensioned matrices $N$, $M, F$, and $H$; and two positive scalars $\kappa>0, \kappa_{d}>0$ such that the following LMIs hold:

$$
\begin{gathered}
\left(\begin{array}{cc}
\Xi & -\tau_{2} \widehat{M} \\
* & -\tau_{2} W_{1}
\end{array}\right)<0, \\
\left(\begin{array}{cc}
\Xi & -\left(\tau_{2}-\tau_{1}\right) \widehat{N} \\
* & -\left(\tau_{2}-\tau_{1}\right)\left(W_{1}+W_{2}\right)
\end{array}\right)<0, \\
\left(\begin{array}{cc}
\Xi & -\left(\tau_{2}-\tau_{1}\right) \widehat{F} \\
* & -\left(\tau_{2}-\tau_{1}\right) W_{2}
\end{array}\right)<0,
\end{gathered}
$$

where

$$
\begin{aligned}
& \Xi=\left(\begin{array}{cccccc}
\Xi_{11} & \Xi_{12} & P D-C^{T} & \Xi_{14} & A^{T} H & \Xi_{16} \\
* & \Xi_{22} & -C_{d}^{T} & 0 & A_{d}^{T} H & \Xi_{26} \\
* & * & -\gamma I & 0 & D^{T} H & 0 \\
* & * & * & \Xi_{44} & \Xi_{45} & 0 \\
* & * & * & * & \Xi_{55} & 0 \\
* & * & * & * & * & \Xi_{66}
\end{array}\right), \\
& \Xi_{16}=\left(\begin{array}{ll}
\Theta_{1} P & 0
\end{array}\right), \quad \Xi_{26}=\left(\begin{array}{ll}
0 & \Theta_{2} P
\end{array}\right), \\
& \Xi_{66}=\operatorname{diag}\{-P,-P\}, \quad \widehat{N}=\left(N^{T}, 0_{1 \times 9}\right)^{T}, \\
& \widehat{M}=\left(M^{T}, 0_{1 \times 9}\right)^{T}, \quad \widehat{F}=\left(F^{T}, 0_{1 \times 9}\right)^{T}, \\
& \Xi_{11}, \Xi_{12}, \Xi_{14}, \Xi_{22}, \Xi_{44}, \Xi_{45}, \Xi_{55}
\end{aligned}
$$

defined in Theorem 11 .

\section{Dissipative Delay-Feedback Control for NSDSs}

Extending on the results of the foregoing section, our aim is to develope an LMIs-based solution to the problem of designing a delay-feedback controller as

$$
u(t, t-\tau(t))=K_{0} x(t)+K_{1} x(t-\tau(t)),
$$

which will render the NSDSs (10) strictly $(Q, S, R)$-dissipative. The closed-loop systems is now described by

$$
\begin{gathered}
\mathrm{d} x(t)=\left\{\widetilde{A} x(t)+f(t, x(t))+\widetilde{A}_{d} x(t-\tau(t))\right. \\
\left.+f_{d}(t, x(t-\tau(t)))+D v(t)\right\} \mathrm{d} t \\
+G(t, x(t), x(t-\tau(t))) \mathrm{d} \omega(t), \\
z(t)=C x(t)+C_{d} x(t-\tau(t)), \\
x(t)=\varphi(t), \quad t \in\left[-\tau_{2}, 0\right),
\end{gathered}
$$

where $\widetilde{A}=A+B K_{0}, \widetilde{A}_{d}=A_{d}+B K_{1}$.

Applying Theorem 7, together with Lemma 5 and congruent transformation, we can get the following theorem without detailed proofs.

Theorem 14. Consider the NSDSs (50). Given some scalars $\alpha>0, \beta>0, \beta_{d}>0, \kappa>0$, and $\kappa_{d}>0$ and matrices $Q=Q^{T} \leq 0, R=R^{T}>0$, and $S$, suppose there exist matrices $X=X^{T}>0, W=W^{T}>0, Y$, and $Y_{d}$ such that the following LMI holds:

$$
\left(\begin{array}{cccc}
\Gamma_{1} & A_{d} X+B Y_{d} & D-X C^{T} S & \Gamma_{2} \\
* & -\widetilde{W} & -X C_{d}^{T} S & \Gamma_{3} \\
* & * & -R_{\alpha} & 0 \\
* & * & * & \Gamma_{4}
\end{array}\right)<0
$$


then the NSDSs (50) are strictly $(Q, S, R)$-dissipative independent of delay; the feedback gain is $K_{0}=Y X^{-1}, K_{1}=$ $Y_{d} X^{-1}$, where $\Gamma_{1}=X A^{T}+A X+Y^{T} B^{T}+B Y+\widetilde{W}$, $\Gamma_{2}=\left(\begin{array}{lllllll}I & I & X C^{T} Q & X \Theta_{1} & 0 & \kappa \beta X & 0\end{array}\right), \quad \Gamma_{3}=$ $\left(\begin{array}{lllllll}0 & 0 & X C_{d}^{T} Q & 0 & X \Theta_{2} & 0 & \kappa_{d} \beta_{d} X\end{array}\right)$, and $\Gamma_{4}=\operatorname{diag}\left(-\kappa I,-\kappa_{d} I\right.$, $\left.\mathrm{Q},-X,-X,-\kappa I,-\kappa_{d} I\right)$.

Similarly, applying Theorem 11, together with Lemma 5 and congruent transformation, we can obtain the following theorem without detailed proofs.

Theorem 15. Consider the NSDSs (50). For the given scalars $\alpha>0, \tau_{2}>\tau_{1} \geq 0, \tau_{d}>0, \kappa>0, \kappa_{d}>0, \beta>0$, and $\beta_{d}>0$, the NSDSs (50) are strictly $(Q, S, R)$-dissipative for all time-varying delays and the feedback gain is $K_{0}=Y X^{-1}, K_{1}=$ $Y_{d} X^{-1}$, if there exist symmetric positive-definite matrices $X, \widetilde{V}_{1}$, $\widetilde{V}_{2}, \widetilde{V}_{3}, \widetilde{W}_{1}, \widetilde{W}_{2}, \widetilde{Z}_{1}$, and $\widetilde{Z}_{2}$ and any appropriately dimensioned matrices $\widetilde{N}, \widetilde{M}, \widetilde{F}, Y$, and $Y_{d}$ such that the following LMIs hold:

$$
\begin{gathered}
\left(\begin{array}{cc}
\Delta & -\tau_{2} \check{M} \\
* & -\tau_{2} \widetilde{W}_{1}
\end{array}\right)<0, \\
\left(\begin{array}{cc}
\Delta & -\left(\tau_{2}-\tau_{1}\right) \check{N} \\
* & -\left(\tau_{2}-\tau_{1}\right)\left(\widetilde{W}_{1}+\widetilde{W}_{2}\right)
\end{array}\right)<0, \\
\left(\begin{array}{cc}
\Delta & -\left(\tau_{2}-\tau_{1}\right) \check{F} \\
* & -\left(\tau_{2}-\tau_{1}\right) \widetilde{W}_{2}
\end{array}\right)<0,
\end{gathered}
$$

where

$$
\begin{aligned}
& \Delta=\left(\begin{array}{cccccc}
\Delta_{11} & \Delta_{12} & \Delta_{13} & \Delta_{14} & \Delta_{15} & \Delta_{16} \\
* & \Delta_{22} & -X C_{d}^{T} S & 0 & \Delta_{25} & \Delta_{26} \\
* & * & -R_{\alpha} & 0 & D^{T} & 0 \\
* & * & * & \Delta_{44} & \Delta_{45} & 0 \\
* & * & * & * & \Delta_{55} & 0 \\
* & * & * & * & * & \Delta_{66}
\end{array}\right), \\
& \Delta_{11}=A X+B Y+X A^{T}+Y^{T} B^{T}+\widetilde{V}_{1}+\widetilde{V}_{2}+\widetilde{V}_{3}+\tau_{2} \widetilde{Z}_{1} \\
& +\left(\tau_{2}-\tau_{1}\right) \widetilde{Z}_{2}+\widetilde{M}+\widetilde{M}^{T} \\
& \Delta_{12}=\widetilde{N}-\widetilde{F}-\widetilde{M}+A_{d} X+B Y_{d}, \quad \Delta_{13}=D-X C^{T} S \\
& \Delta_{14}=\left(\begin{array}{llll}
\widetilde{F} & -\widetilde{N} & I & I
\end{array}\right), \quad \Delta_{15}=Y^{T} B^{T}+X A^{T}, \\
& \Delta_{16}=\left(\begin{array}{lllll}
X C^{T} Q & X \Theta_{1} & 0 & \kappa \beta X & 0
\end{array}\right), \\
& \Delta_{22}=-\left(1-\tau_{d}\right) \widetilde{V}_{2} \\
& \Delta_{25}=Y_{d}^{T} B^{T}+X A_{d}^{T} \\
& \Delta_{26}=\left(\begin{array}{lllll}
X C_{d}^{T} Q & 0 & X \Theta_{2} & 0 & \kappa_{d} \beta_{d} X
\end{array}\right),
\end{aligned}
$$

$$
\begin{gathered}
\Delta_{44}=\operatorname{diag}\left\{-\widetilde{V}_{1},-\widetilde{V}_{3},-\kappa I,-\kappa_{d} I\right\}, \\
\Delta_{45}=\left(\begin{array}{llll}
0 & 0 & I & I
\end{array}\right)^{T}, \\
\Delta_{55}=\tau_{2} \widetilde{W}_{1}+\left(\tau_{2}-\tau_{1}\right) \widetilde{W}_{2}-X-X^{T}, \\
\Delta_{66}=\operatorname{diag}\left\{Q,-X,-X,-\kappa I,-\kappa_{d} I\right\}, \\
\check{N}=\left(\widetilde{N}^{T}, 0_{1 \times 12}\right)^{T}, \quad \check{M}=\left(\widetilde{M}^{T}, 0_{1 \times 12}\right)^{T}, \\
\check{F}=\left(\widetilde{F}^{T}, 0_{1 \times 12}\right)^{T} .
\end{gathered}
$$

\section{Numerical Example with Simulation}

In this section, we will give an example to show the correctness of the derived results and the effectiveness of the designed controller. Consider the following nonlinear stochastic delay systems:

$$
\begin{aligned}
\mathrm{d} x(t)= & {\left[\left(\begin{array}{cccc}
-0.1 & 1 & 0 & 1 \\
2 & -1 & 2.5 & 0 \\
-1 & -1.5 & -5 & 0 \\
0 & 0 & 2 & -5
\end{array}\right) x(t)\right.} \\
& +\left(\begin{array}{c}
0.1 x_{1} \sin \left(x_{3}\right) \\
0.4 x_{2} \cos \left(x_{4}\right) \\
0.3 x_{3} \sin \left(x_{1} x_{4}\right) \\
0.5 x_{4} \cos \left(x_{2} x_{3}\right)
\end{array}\right)
\end{aligned}
$$$$
+\left(\begin{array}{cccc}
-1 & 1 & 0 & 1 \\
2 & -3 & 2.5 & 0 \\
-1 & -2 & -3 & 0 \\
0 & 0 & 2 & 3
\end{array}\right) x(t-\tau)
$$$$
+\left(\begin{array}{c}
0.1 x_{1}(t-\tau) \sin \left(x_{3}(t-\tau)\right) \\
0.2 x_{2}(t-\tau) \cos \left(x_{4}(t-\tau)\right) \\
0.3 x_{3}(t-\tau) \sin \left(x_{1}(t-\tau) x_{4}(t-\tau)\right) \\
0.3 x_{4}(t-\tau) \cos \left(x_{2}(t-\tau) x_{3}(t-\tau)\right)
\end{array}\right)
$$$$
+\left(\begin{array}{cc}
0 & -1 \\
-0.5 & 0 \\
-0.2 & 0 \\
0 & -0.2
\end{array}\right) u(t, t-\tau)
$$$$
\left.+\left(\begin{array}{cccc}
0.5 & 0.1 & 0.1 & 0 \\
0.1 & 0.1 & 0.5 & 0.2 \\
0 & 0.1 & 0 & 1 \\
0 & 0 & 1 & 0.4
\end{array}\right) v(t)\right] \mathrm{d} t
$$

$$
+\left(\begin{array}{c}
0.35 x_{1} \sin \left(x_{1}\right) \\
+0.35 x_{1}(t-\tau) \\
\times \cos \left(x_{2}(t-\tau)\right) \\
\frac{0.71 t}{1+t}\left(x_{2}+x_{2}(t-\tau)\right) \\
0.35 x_{3} \sin \left(x_{1} x_{3}\right) \\
-0.35 x_{3}(t-\tau) \\
\times \sin \left(x_{3}^{2}(t-\tau)\right) \\
\frac{14 t}{7+100 t}\left(x_{4}-x_{4}(t-\tau)\right)
\end{array}\right) \mathrm{d} \omega(t)
$$




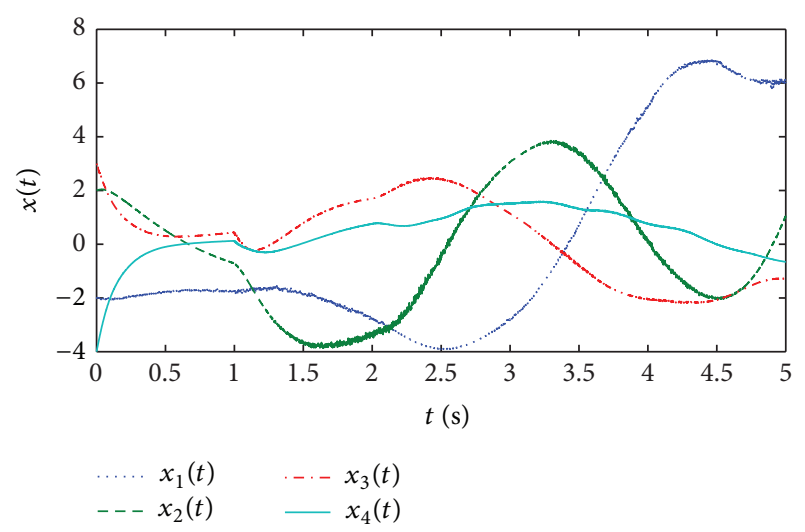

FIGURE 1: The states curves of open-loop NSDSs in (54) without the control with initial state $(-2,2,3,-4)^{T}$ and time delay $\tau=1$.

$$
\begin{aligned}
z(t)= & \left(\begin{array}{cccc}
1 & 2 & 0 & 0 \\
0 & 0.5 & 0 & 0 \\
0 & 0 & 1 & 0 \\
0 & 0 & 0.2 & 1
\end{array}\right) x(t) \\
& +\left(\begin{array}{cccc}
1 & 1 & 0 & -1 \\
0.5 & 0.5 & -1 & 0 \\
0 & 0 & 1 & 2 \\
0 & 0 & 2 & -1
\end{array}\right) x(t-\tau)
\end{aligned}
$$

For Case 1, when the initial condition $x(0)=[-2,2,3$, $-4]^{T}$ is used and $v(t)$ is a random vector of zero mean and 0.3 standard deviation, we can see that the uncontrolled NSDSs (54) are not stable from Figure 1. Hence the design of dissipative delay-feedback controller is necessary. From (54), we can get $\Theta_{1}=\Theta_{2}=\operatorname{diag}(0.5,1,0.5,2)$, and $\beta=0.5$, $\beta_{d}=0.3$, for given $S=I, R=5 I$, and $Q=-0.2 I$; applying Theorem 14 to this example, we have

$$
\begin{aligned}
X= & \left(\begin{array}{cccc}
38.2649 & -9.3804 & -3.6924 & 7.8006 \\
-9.3804 & 25.9896 & 10.5274 & -1.9189 \\
-3.6924 & 10.5274 & 4.5464 & -0.7337 \\
7.8006 & -1.9189 & -0.7337 & 2.2634
\end{array}\right), \\
Y= & 1.0 e+004 \\
& *\left(\begin{array}{cccc}
-0.0254 & 1.5597 & 0.6249 & -0.0044 \\
0.8881 & -0.0450 & -0.0175 & 0.1783
\end{array}\right), \\
Y_{d}= & 1.0 e+003 \\
& *\left(\begin{array}{cccc}
1.1951 & 0.5087 & 0.2100 & 0.2313 \\
0.0308 & 0.2403 & 0.0994 & -0.0020
\end{array}\right) .
\end{aligned}
$$

So the delay-feedback controller parameters can be calculated as follows:

$$
\begin{gathered}
K=\left(\begin{array}{cccc}
149.4677 & 780.3725 & -306.7689 & 27.4064 \\
258.0621 & 100.2942 & -67.4539 & -38.6640
\end{array}\right), \\
K_{d}=\left(\begin{array}{cccc}
43.1863 & 33.7078 & 0.3173 & -17.9504 \\
5.8953 & 7.6550 & 6.9022 & -12.4934
\end{array}\right) .
\end{gathered}
$$

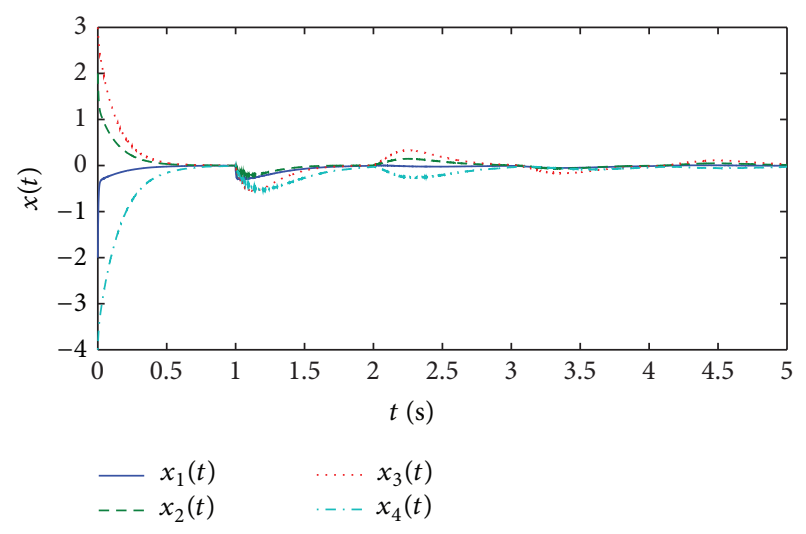

FIGURE 2: The states curves of closed-loop NSDSs in (54) under the delay-feedback control with initial state $(-2,2,3,-4)^{T}$ and time delay $\tau=1$.

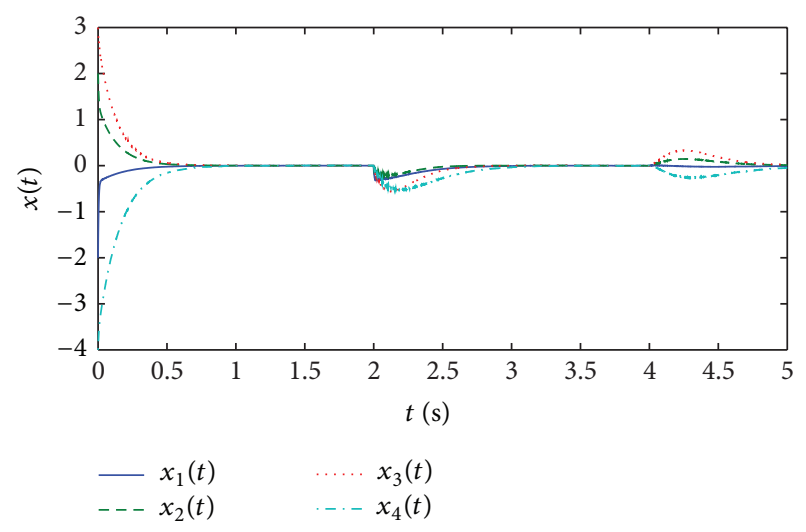

FIGURE 3: The states curves of closed-loop NSDSs in (54) under the delay-feedback control with initial state $(-2,2,3,-4)^{T}$ and time delay $\tau=2$.

The states curves and the output curves of closed-loop NSDSs in (54) can be seen in Figures 2, 3, and 4; from Figure 5, we can see that (12) holds. Hence, the closed-loop NSDSs are strictly $(Q, S, R)$-dissipative; we can also see that the delay-feedback controller is delay-independent.

\section{Conclusions}

The dissipative delay-feedback control problems for nonlinear stochastic delay systems (NSDSs) have been investigated based on dissipativity analysis. The systems are subjected to stochastic disturbance, nonlinear disturbance, and two cases time-delay effects, which often exist in a wide variety of industrial processes and are the main sources of instability. Based on the Lyapunov stability theory and stochastic analysis technique, both delay-independent and delay-dependent dissipativity criteria have been established in terms of linear matrix inequalities (LMIs). The available results on $H_{\infty}$ approach and passivity for stochastic delay systems as special cases of the developed results also have been given in this paper. The delay-dependent feedback controller has been 


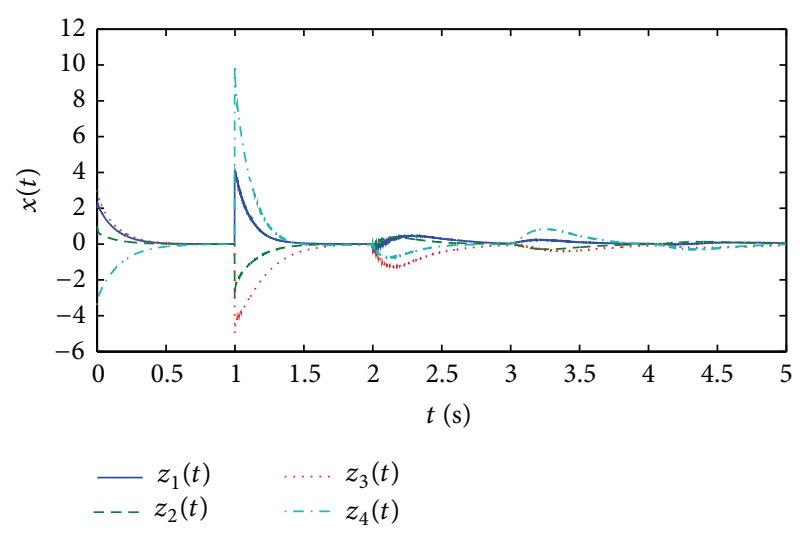

Figure 4: The output curves of closed-loop NSDSs in (54) under the delay-feedback control with initial state $(-2,2,3,-4)^{T}$ and time delay $\tau=1$.

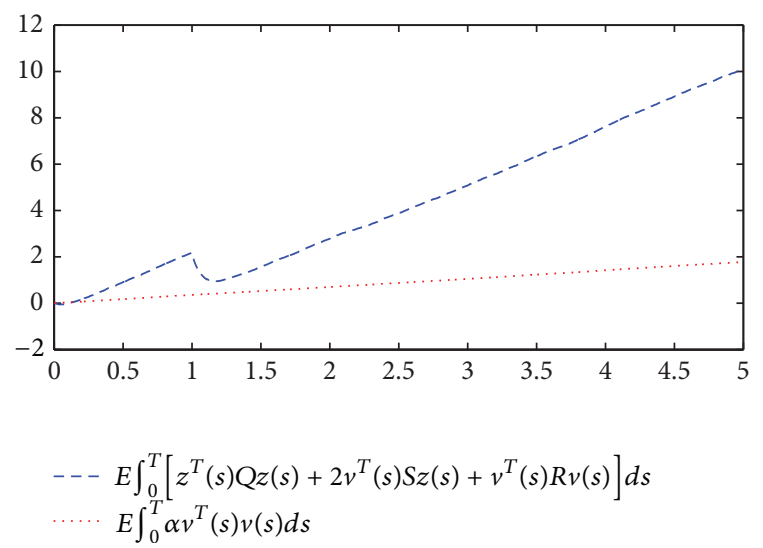

FIGURE 5: The dissipativity level of closed-loop NSDSs in (54) under the delay-feedback control with initial state $(-2,2,3,-4)^{T}$ and time delay $\tau=1$.

designed by considering the relationship among the timevarying delay, its lower and upper bound, and its difference without ignoring any terms, which effectively reduces the conservative. A numerical example also has been given to verify the effectiveness of the proposed methods.

\section{Conflict of Interests}

The authors declare that there is no conflict of interests regarding the publication of this paper.

\section{Acknowledgments}

This work is supported by the National Science Foundation of China (NSFC) under Grants nos. 61104127, 51079057, and 61134012; China Postdoctoral Science Foundation under Grant no. 2012M521428; and Hubei Province Key Laboratory of Systems Science in Metallurgical Process (Wuhan University of Science and Technology) under Grant no. Y201320.

\section{References}

[1] R. Z. Khaminskii, Stochastic Stability of Differential Equations, vol. 7, Sijthoff \& Noordhoff, Alphen aan den Rijn, The Netherlands, 1980.

[2] Y. Shen and J. Wang, "An improved algebraic criterion for global exponential stability of recurrent neural networks with timevarying delays," IEEE Transactions on Neural Networks, vol. 19, no. 3, pp. 528-531, 2008.

[3] Y. Shen and J. Wang, "Robustness analysis of global exponential stability of recurrent neural networks in the presence of time delays and random disturbances," IEEE Transactions on Neural Networks and Learning Systems, vol. 23, no. 1, pp. 87-96, 2012.

[4] G. Chen and Y. Shen, "Robust reliable $H_{\infty}$ control for nonlinear stochastic Markovian jump systems," Mathematical Problems in Engineering, vol. 2012, Article ID 431576, 16 pages, 2012.

[5] Y. Shen and J. Wang, "Noise-induced stabilization of the recurrent neural networks with mixed time-varying delays and Markovian-switching parameters," IEEE Transactions on Neural Networks, vol. 18, no. 6, pp. 1857-1862, 2007.

[6] Y. Shen and J. Wang, "Almost sure exponential stability of recurrent neural networks with Markovian switching," IEEE Transactions on Neural Networks, vol. 20, no. 5, pp. 840-855, 2009.

[7] Q. Luo, X. Mao, and Y. Shen, "New criteria on exponential stability of neutral stochastic differential delay equations," Systems \& Control Letters, vol. 55, no. 10, pp. 826-834, 2006.

[8] C. Wang and Y. Shen, "Improved delay-dependent robust stability criteria for uncertain time delay systems," Applied Mathematics and Computation, vol. 218, no. 6, pp. 2880-2888, 2011.

[9] W. Zhang and L. Xie, "Interval stability and stabilization of linear stochastic systems," IEEE Transactions on Automatic Control, vol. 54, no. 4, pp. 810-815, 2009.

[10] W. Zhang, B.-S. Chen, and C.-S. Tseng, "Robust $H_{\infty}$ filtering for nonlinear stochastic systems," IEEE Transactions on Signal Processing, vol. 53, no. 2, part 1, pp. 589-598, 2005.

[11] B.-S. Chen and W. Zhang, "Stochastic $H_{2} / H_{\infty}$ control with state-dependent noise," IEEE Transactions on Automatic Control, vol. 49, no. 1, pp. 45-57, 2004.

[12] W. Zhang, Y. Huang, and H. Zhang, "Stochastic $H_{2} / H_{\infty}$ control for discrete-time systems with state and disturbance dependent noise," Automatica, vol. 43, no. 3, pp. 513-521, 2007.

[13] J. C. Willems, "Dissipative dynamical systems. I: general theory," Archive for Rational Mechanics and Analysis, vol. 45, pp. 321-351, 1972.

[14] D. Hill and P. Moylan, "The stability of nonlinear dissipative systems," IEEE Transactions on Automatic Control, vol. 21, no. 5, pp. 708-711, 1976.

[15] Z. Q. Tan, Y. C. Soh, and L. Xie, "Dissipative control for linear discrete-time systems," Automatica, vol. 35, no. 9, pp. 1557-1564, 1999.

[16] M. S. Mahmoud, "Delay-dependent dissipativity analysis and synthesis of switched delay systems," International Journal of Robust and Nonlinear Control, vol. 21, no. 1, pp. 1-20, 2011.

[17] M. S. Mahmoud, Y. Shi, and F. M. AL-Sunni, "Dissipativity analysis and synthesis of a class of nonlinear systems with timevarying delays," Journal of the Franklin Institute: Engineering and Applied Mathematics, vol. 346, no. 6, pp. 570-592, 2009.

[18] K. Youcef-Toumi and O. Itô, "A time delay controller for systems with unknown dynamics," Journal of Dynamic Systems, Measurement, and Control, vol. 112, no. 1, pp. 133-142, 1990. 
[19] V. Kapila, A. Tzes, and Q. Yan, "Closed-loop input shaping for flexible structures using time-delay control," Journal of Dynamic Systems, Measurement and Control, vol. 122, no. 3, pp. 454-460, 2000.

[20] X. Mao, J. Lam, and L. Huang, "Stabilisation of hybrid stochastic differential equations by delay feedback control," Systems \& Control Letters, vol. 57, no. 11, pp. 927-935, 2008.

[21] D. J. Hill and P. J. Moylan, "Dissipative dynamical systems: basic input-output and state properties," Journal of the Franklin Institute, vol. 309, no. 5, pp. 327-357, 1980.

[22] S. Boyd, L. El Ghaoui, E. Feron, and V. Balakrishnan, Linear Matrix Inequalities in System and Control Theory, vol. 15, SIAM, Philadelphia, Pa, USA, 1994.

[23] K. Gu, "An integral inequality in the stability problem of timedelay systems," in Proceedings of the 39th IEEE Confernce on Decision and Control, pp. 2805-2810, Sydney, Australia, December 2000 . 


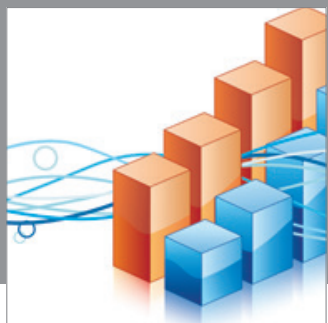

Advances in

Operations Research

mansans

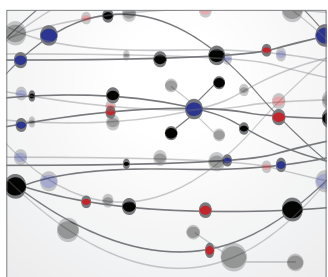

The Scientific World Journal
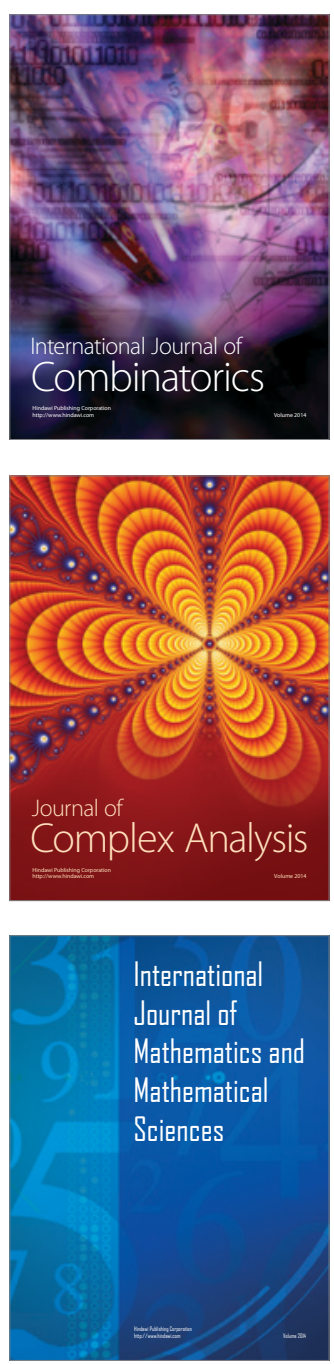
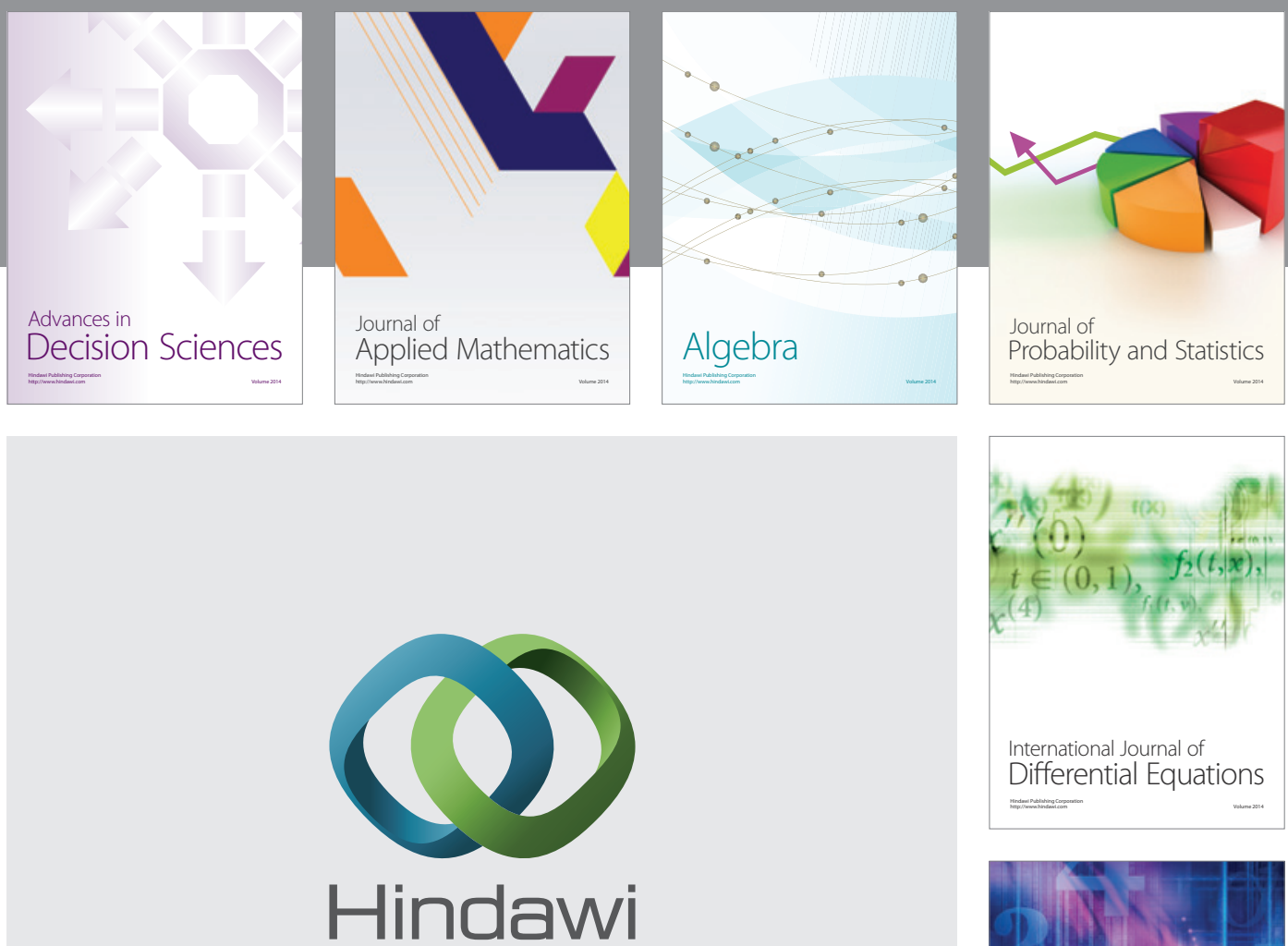

Submit your manuscripts at http://www.hindawi.com
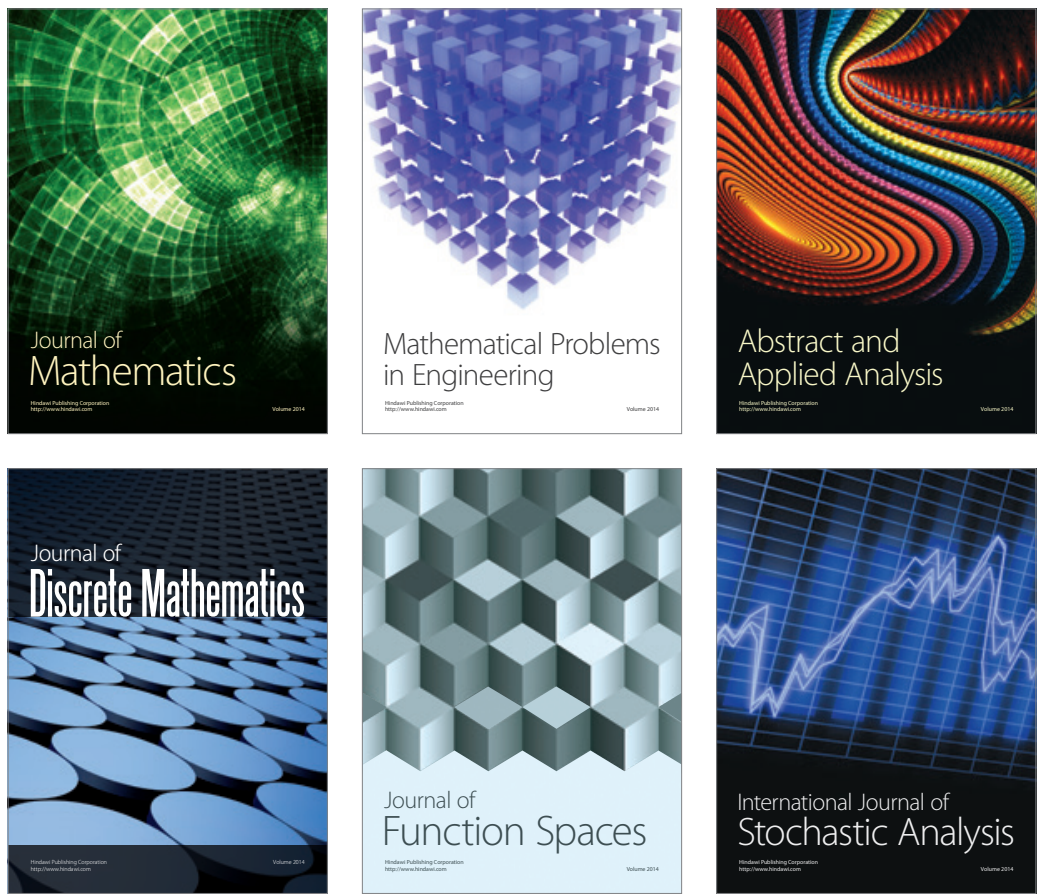

Journal of

Function Spaces

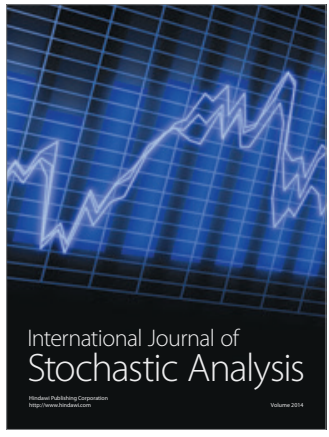

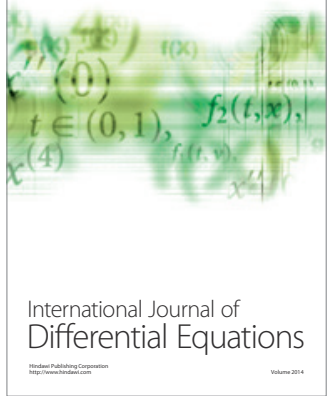
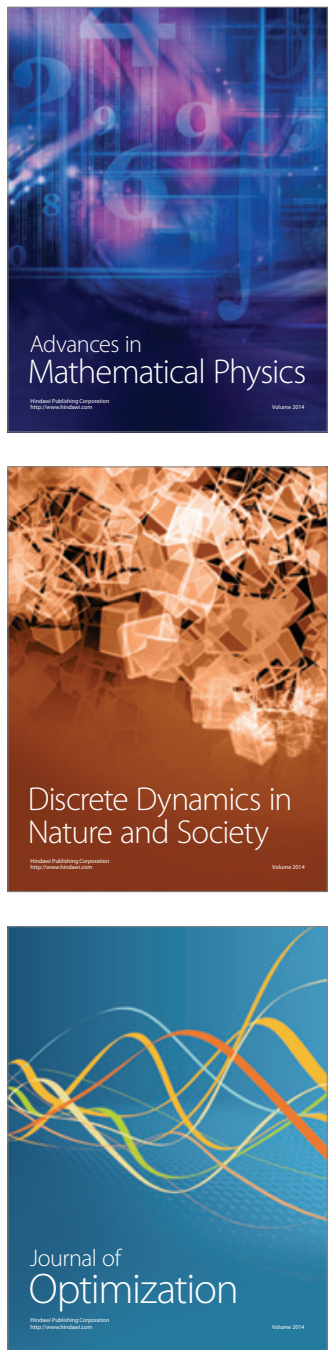